Conclusion Only one CDR developed for children with FN met all methodological standards and reached the highest level of evidence.

\section{PS-090 HEREDITARY SPHEROCYTOSIS AND RED CELL INDICES MCHC, MCV, RDW}

${ }^{1} \mathrm{~A}$ Barbullushi, ${ }^{1} \mathrm{P}$ Daja, ${ }^{2} \mathrm{D}$ Bali, ${ }^{2} \mathrm{~N}$ Maliqari, ${ }^{3} \mathrm{~A}$ Godo. 'Laboratory Departament, Universitary Hospital "Mather Tereza", Tirane, Albania; ${ }^{2}$ Pediatric Departament, Universitary Hospital "Mather Tereza", Tirane, Albania; "Pediatric Departament, Universitary Hospital "Mather Tereza", Tirane, Albania

\subsection{6/archdischild-2014-307384.384}

Background Hereditary spherocytosis (HS) is a common inherited disorder that is characterised by anaemia, jaundice, and splenomegaly. Clinical severity is variable with most patients having a well-compensated hemolytic anaemia. The primary lesion in HS is loss of membrane surface area, leading to reduced deformability due to defects in the membrane proteins ankyrin, band 3, beta spectrin, alpha spectrin, or protein 4.2. Many isolated mutations have been identified in the genes encoding these membrane proteins; common hereditary spherocytosis-associated mutations have not been identified. The classic laboratory features of HS include minimal or no anaemia, reticulocytosis, an increased mean corpuscular haemoglobin concentration (MCHC), spherocytes on the peripheral blood smear, hyperbilirubinemia, and abnormal results on the osmotic fragility test.

Aim Of the study is to evaluate the role of MCV, MCHC as a screen test to diagnose spherocytosis

Methods In our study are included 60 subjects, 30 children with HS and 30 children-control groups. Our patients with anaemia, jaundice, and splenomegaly are diagnose with HS by incubated osmotic fragility test, performed after incubating RBCs for 18$24 \mathrm{~h}$ under sterile conditions at $37^{\circ} \mathrm{C}$.

Results We found that 25\% of pts. have mild HS, 20\% moderate HS, $30 \%$ moderate to severe HS and $25 \%$ severe HS. In peripheral blood smear $7 \%$ of pts. had $0-5$ spherocites for field, $30 \%$ had 5-10 spherocites for field and 63\% had 10-15 spherocites for field. $70 \%$ of pts. With HS have MCHC $>38 \%$.

There are a positive correlation between MCHC and spherocites in peripheral blood smear $(\mathrm{r}=0,898, \mathrm{p}<0,001)$ and RDW $(\mathrm{r}=0,647, \mathrm{p}<0.001)$, negative correlation between MCHC and MCV ( $\mathrm{r}=-0,437 \mathrm{p}<0,001)$

Conclusion The dedication of hiperdense erythrociyte today is used as a new tool in diagnosing HS. The determination of MCHC constantly growing with other red cell index, MCV < $80 \mathrm{fl}$, RDW > 15 obtained from an electronic cell counter usually is enough to suggest for HS.

Key Words Spherocytosis, MCHC, anaemia, children.

\section{PS-091 DISPERSION OF THE QT AND QTC INTERVALS-EARLY MARKER OF ANTHRACYCLINE INDUCED CARDIOTOXICITY IN CHILDREN WITH MALIGNANT HEMOPATHIES}

${ }^{1} \mathrm{~L}$ Dimitriu, ${ }^{2} \mathrm{AG}$ Dimitriu, ${ }^{3} \mathrm{I}$ Miron, ${ }^{4} \mathrm{C}$ Mandric. ${ }^{1}$ Pediatric Cardiology, Medex Medical Center, lasi, Romania; ${ }^{2}$ Pediatric Cardiology, University of Medicine and Pharmacy, lasi, Romania; ${ }^{3}$ Pediatric Hematology-Oncology, University of Medicine and Pharmacy, lasi, Romania; ${ }^{4}$ Pediatrics, Children's Hospital, lasi, Romania

10.1136/archdischild-2014-307384.385

Background Heart rhythm disorders are one of major adverse effects induced by myocardial anthracicline cardiotoxicity in children with malignancies and that require early diagnosis for effective prevention.

Objectives To investigate the utility of the study of QT and QTc dispersion in children with malignancies treated with anthracyclines.

Methods patients: 40 patients (2-18 years) with malignant hemopathies, treated with anthracyclines. All patients were examined by clinical examen, ECG, Doppler echocardiography, the values of QT dispersion (difference between the maximum and minimum QT interval, manually measure the QT interval, on three successive cardiac cycles) and QT dispersion (Bazett's formula). Dispersion of QT and QTc interval in these patients was compared to similar values from 20 healthy children without cardiovascular history.

Results The increase of QT and QTc dispersion in patients comparative to the control lot, was revealed in $73 \%$ cases, usually in those which had a cumulative anthracyclines doses over $400 \mathrm{mg} /$ $\mathrm{m}^{2}$, with medium values of QTD: $53,33 \pm 10,18 \mathrm{msec}$ and QTcD: 66,28 $\pm 12,8$ msec. The increased dispersion of QT and QTc intervals was highlight most frequently in cases with echocardiographycal signs of anthracyclines cardiotoxicity, even only diastolic dysfunction of left ventricle.

Conclusions The significant incidence of increasing the QT and QTc interval dispersion in patients who received treatment with anthracyclines and the correlation with cumulative anthracyclines doses and echocardiographic modifications, especially diastolic dysfunction, proves utility of systematic investigation of QT and QTc intervals dispersion in the full control in the therapy, as an earlier marker for cardiotoxicity of anthracyclines.

\section{PS-092 NUTRITIONAL STATUS OF CHILDREN DIAGNOSED WITH ACUTE LYMPHOBLASTIC LEUKAEMIA AT THE CHILDREN CANCER CENTRE}

${ }^{1} \mathrm{~N}$ Yazbeck, ${ }^{2} \mathrm{~L}$ Samia, ${ }^{3} \mathrm{R}$ Saab, ${ }^{3} \mathrm{MR}$ Abboud, ${ }^{3} \mathrm{~S}$ Muwakkit. ${ }^{1}$ Department of Pediatrics and Adolescent Medicine, American University of Beirut - Medical Center, Beirut, Lebanon; ${ }^{2}$ Department of Pediatrics and Adolescent Medicine Fellow Children Cancer Center of Lebanon, American University of Beirut Medical Center, Beirut, Lebanon; ${ }^{3}$ Department of Pediatrics and Adolescent Medicine Children Cancer Center of Lebanon, American University of Beirut Medical Center, Beirut, Lebanon

\subsection{6/archdischild-2014-307384.386}

Background and aims Acute lymphoblastic leukaemia (ALL) is the most common malignancy among children. Malnutrition remains a major concern for paediatric oncologists. Although studies have shown that malnutrition can negatively affect treatment outcome, results are still controversial. This retrospective cohort study aims at determining the prevalence of malnutrition and its association with treatment outcome and infection among children with ALL treated at the Children Cancer Centre in Lebanon (CCCL).

Methods 108 children and adolescents diagnosed with ALL between April 2002 and May 2010 were enrolled in the study. Anthropometric data were collected from patient's medical record upon diagnosis, at 3 and 6 months, and at the end of treatment. Body mass index (BMI) was calculated for children $\geq$ 2 years while weight for height ratio was used for patients $<2$ years. Patients were considered underweight, stunted, or wasted if their z-scores were <-2SD.

Results The prevalence of malnourished children was $27 \%$ at diagnosis and remained almost the same at the end of treatment. The odds ratio of having worse outcome in terms of relapse or death was higher among malnourished children with OR = $2.09,95 \% \mathrm{CI}=0.3-13.4$ and $\mathrm{OR}=1.25$ and $95 \% \mathrm{CI}=0.2-$ 6.9 for death and relapse respectively. However this trend was 
not statistically significant. Concomitantly, a slight increase in the odds of having positive bacterial bloodor urinary culture was observed among malnourished children without statistical significance $(\mathrm{OR}=1.35,95 \% \mathrm{CI}=0.4-3.7)$.

Conclusion Although there was a trend showing worse outcomes in malnourished children with ALL larger studies need to be conducted to prove the association.

\section{PS-093 EXOSOMES FROM MYCN-AMPLIFIED NEUROBLASTOMA CELLS CONTAIN ONCOGENIC MIRNAS}

T Flægstad, BH Haug, OH Hald, P Utnes, C Løkke, C Einvik. Pediatrics, Institute of Clinical Medicine, Tromso, Norway

\subsection{6/archdischild-2014-307384.387}

The MYCN oncogene is frequently amplified in neuroblastoma and is associated with aggressive disease and treatment failure. MYCN induces expression of different miRNAs, which are posttranscriptional regulators of gene expression with established roles in neuroblastoma development.

In this study, we show that MYCN -amplified cell lines not only express miRNAs with functions inside the cell, but also secrete populations of miRNAs inside small vesicular structures called exosomes, with the ability to shuttle to other cells. We have isolated and characterised exosomes from MYCN amplified cell lines, and here demonstrate their ability to be taken up by other cells. By profiling the miRNA expression, we demonstrate high expression of a group of established on comirs in exosomes from two MYCN- amplified cell lines. Despite the fact that other studies have demonstrated the ability of exosomal miRNAs to regulate their targets in recipient cells, we did not observe this with exosomes from MYCN -amplified cells. However, exosomes were able to induce in vitro angiogenic tube formation, suggesting that they can have tumorigenic properties.

These new findings reveal a potential new way for MYCN amplified neuroblastoma cells to interact with their environment.

\section{PS-094 THE LEVELS OF BONE ALKALINE PHOSPHATASE AND HUMAN EPIDERMAL GROWTH FACTOR RECEPTOR-2 IN PATIENTS WITH OSTEOSARCOMA}

1J Gajewska, ${ }^{1} \mathrm{~J}$ Ambroszkiewicz, ${ }^{1} \mathrm{M}$ Chelchowska, ${ }^{2} \mathrm{~T}$ Klepacka, ${ }^{3} \mathrm{M}$ RychlowskaPruszynska. 'Screening Department, Mother and Child Institute, Warsaw, Poland; ${ }^{2}$ Department of Pathomorphology, Mother and Child Institute, Warsaw, Poland; ${ }^{3}$ Department of Oncological Surgery for Children and Youth, Mother and Child Institute, Warsaw, Poland

\subsection{6/archdischild-2014-307384.388}

Background and aims Osteosarcoma (OS) is a primary malignant bone tumour, deriving from primitive bone-forming mesenchyme and characterised by the production of osteoid tissue or immature bone. The importance of bone alkaline phosphatase (BALP) and human epidermal growth factor receptor-2 (HER-2) as prognostic markers is still discussed. The aim of this study was to investigate serum levels of BALP and HER-2 in patients with OS during clinical treatment.

Methods We studied 22 patients (5 girls, 17 boys) at the age 7-20 years with osteosarcoma treated at the Institute of Mother and Child in Warsaw. All patients were evaluated for the serum levels of BALP and HER-2 before treatment onset, during pre- and postoperative chemotherapy and after termination of treatment. Healthy children $(\mathrm{n}=22)$ were the reference group. The levels of BALP and HER-2 were measured using immunoenzymatic methods.
Results The values of BALP and HER-2 proteins were higher ( $\mathrm{p}$ $<0.01 ; \mathrm{p}<0.05)$ in patients with OS at diagnosis compared with the control group. The values of both markers significantly decreased during chemotherapy in most patients with remission. After therapy, the activity of BALP was higher in patients with progression than with remission of disease $(p<0.001)$.

Conclusions Our results demonstrate different pattern of BALP and HER-2 proteins during clinical treatment. However, low values of both markers during therapy may characterise remission of disease and favourable prognosis for patients with OS. Further longitudinal studies are necessary to confirm the prognostic values of BALP and HER-2 proteins in this group of patients.

\section{PS-095 THE ASSESSMENT OF NUTRITIONAL STATUS IN A GROUP OF CHILDREN WITH CANCER FROM ROMANIA}

M Chincesan, O Marginean, A Grama. Paediatrics, University of Medicine and Pharmacy, TG MURES, Romania

\subsection{6/archdischild-2014-307384.389}

Background Despite recent technological advances, inadequate nutrition has been clearly identified as a significant risk factor to survival of paediatric cancer patients; having in view this issue we proposed to assess the nutritional status of children with cancer.

Methods We prospectively assessed 47 consecutively hospitalised patients in a tertiary emergency paediatric hospital. The patients were divided into two groups: Group I (17 patients with cancer) and Group II, the control group consisting of 30 age and gender-matched patients with different paediatric diseases. Weight (W), height $(\mathrm{H})$, body mass index (BMI), middle upper arm circumference (MUAC), tricipital skinfold thickness (TST) were comparatively evaluated. Fat mass (FM), fat free mass (FFM), muscle mass (MM) and total body water (TBW) were measured in the both group using Tanita BC 420SMA Analyzer.

Results Anthropometric parameters in Group I were significantly different from those in Group II for MUAC (-0.10 DS vs 1.31 DS) and TST (-0.01 DS vs 1.35 DS) ( $p=0.01)$. Group I had significantly lower percent FM $(14.79 \pm 7.14$ vs $21.56 \pm$ 9.06; $\mathrm{p}=0.01)$ and significantly higher percent TBW $(61.84 \pm$ 4.93 vs $57.45 \pm 6.64 ; p=0.02)$ compared with Group II. No other significant differences in any body composition components were obtained.

Conclusions Children with cancer had higher alterations of nutritional status. The bioimpedance method provides important information on body composition and allows monitoring of nutritional risk.

Acknowledgment This paper is supported by the project 14/ 30.01.2013: 'The assessment of nutritional status in children with cancer', project financed by The University of Medicine and Pharmacy, Tîrgu-Mures, Romania.

\section{PS-095a THE MEAN PLATELET VOLUME IN INFANTS WITH MECONIUM STAINED AMNIOTIC FLUID}

${ }^{1} \mathrm{M}$ Tekin, ${ }^{1} \mathrm{C}$ Konca, ${ }^{2} \mathrm{Z}$ Kahramaner, ${ }^{2} \mathrm{~A}$ Erdemir, ${ }^{3} \mathrm{~A}$ Gulyuz, ${ }^{4} \mathrm{~F}$ Uckardes, ${ }^{1} \mathrm{M}$ Turgut. ${ }^{1}$ Pediatrics, Adiyaman University School of Medicine, Adiyaman, Turkey; ${ }^{2}$ Pediatrics Neonatology Clinic, Adiyaman University School of Medicine, Adiyaman, Turkey; ${ }^{3}$ Pediatrics, Sevgi Hospital, Malatya, Turkey; ${ }^{4}$ Statistics, Adiyaman University School of Medicine, Adiyaman, Turkey

10.1136/archdischild-2014-307384.390 